\title{
Statistical Texture Features Based Automatic Detection and Classification of Diabetic Retinopathy
}

\author{
Md. Rahat Khan ${ }^{1}$ \\ ${ }^{1}$ Department of Computer Science and Engineering, Khwaja Yunus Ali University, Sirajgonj, Bangladesh \\ Email: rahatkhan.cse@gmail.com \\ A. S. M. Shafi ${ }^{1,2}$ \\ ${ }^{2}$ Department of Computer Science and Engineerin, Mawlana Bhashani Science and Technology University, \\ Tangail, Bangladesh \\ Email: shafi.cse.mbstu11@gmail.com
}

Received: 24 April 2020; Accepted: 06 July 2020; Published: 08 April 2021

\begin{abstract}
Diabetes is a globally prevalent disease that can cause microvascular compilation such as Diabetic Retinopathy (DR) in the human eye organs and it might prompt a significant reason for visual deficiency. The present study aimed to develop an automatic detection and classification system to diagnosing diabetic retinopathy from digital fundus images. An automated diabetic retinopathy detection and classification system from retinal images is proposed in our work to reduce the workload of ophthalmologists. This work comprises three main stages. Our proposed method first extracts the blood vessels from color fundus image. Secondly, the method detects whatever the input image as normal or diabetic retinopathy and then illustrates an automatic diabetic retinopathy classification technique through statistical texture features. It embeds Gray Level Co-occurrence Matrix (GLCM) and Gray Level Run Length Matrix (GLRLM) for second-order and higher-order statistical texture feature as a feature extraction technique into three renowned classifiers namely K-Nearest Neighbor (KNN), Random Forest (RF) and Support Vector Machine (SVM). The evaluation results containing a dataset of 644 retinal images indicate that the proposed method based on random forest classifier is found to be effective with a weighted sensitivity, precision, F1-score and accuracy of $95.53 \% 96.45 \%$, 95.38\% and $95.19 \%$ respectively for the detection and classification of diabetic retinopathy. These outcomes propose, that the method could decrease the cost of screening and diagnosis while achieving higher than suggested performance and that the system could be implemented in clinical assessments requiring better evaluating.
\end{abstract}

Index Terms: Diabetes, Diabetic Retinopathy, Run Length Matrix, Image Classification

\section{Introduction}

Diabetic retinopathy is one of the main causes of global blindness in the economically active population because it affects people between the ages of 20 and 74 [1,2]. It causes other problems like stroke, cardiovascular disease, diabetic nephropathy and diabetic neuropathy. The number of people with diabetic retinopathy is growing higher day by day. This number will estimate to grow from 126.6 million to 191.0 million by 2030 and the number with VisionThreatening Diabetic Retinopathy (VTDR) will grow from 37.3 million to 56.3 million if no appropriate action is taken [3]. The rapid increase of diabetics especially in low-income countries, shortage of expert ophthalmologists and resources, high cost of treatment and time spent on labor-intensive screening are some of the limitations of manual DR detection. This helps in increasing the number of DR patients [4]. Regular eye examination remains the only way for early detection and treatment to prevent vision loss which can reduce the risk by 50\% [5]. There are two types of clinical DR: Non-Proliferative Diabetic Retinopathy (NPDR) or Background Retinopathy and Proliferative Diabetic Retinopathy (PDR). Unfortunately, the DR is usually identified in advanced stages (PDR), with unfavorable forecast even with the right treatment [6]. Therefore, an accurate, automatic, fast and cost-effective technique is needed to detect DR. Although a high sensitivity is good for screening, a relatively low specificity will result in more false-positive cases in the real world [7]. Since 1982, the quantification of diabetic retinopathy and the detection of features such as exudates and blood vessels on fundus images were studied. 
A lot of work has been done in this field. A brief overview of the existing DR detection and classification method has been provided here using various digital image processing techniques. A new approach for routine DR detection by using Discrete Wavelet Transform (DWT) was proposed by Khademi et al. [8]. The method experimented on 38 normal and 48 abnormal images with $79 \%$ specificity, $85.4 \%$ sensitivity and $82.2 \%$ accuracy. Authors [9] presented an unsupervised approach to coarse segmentation for vessel detection with an average accuracy of $87 \%$. They incorporated multiple concepts, i.e., mathematical morphology, curvature and spatial dependency to accurately define thin and elongated vessels from vessel pixels. However, the algorithm was unsuccessful in the determination of vessel diameter and was also found to be less satisfactory at segmenting vessel structures on low contrast images. Sarathi et al. [10] mentioned a real-time approach for Optic Disc (OD) segmentation where the boundary of the disks was extracted after the removal of blood vessels using regionally-adaptive threshold and ellipse fitting methods. The proposed technique achieved significant results when tested on standard databases like MESSIDOR and DRIVE with an average overlapping ratio of $89 \%$ and $87 \%$ respectively. The identification of diabetic retinopathy stage based on Convolution Neural Network (CNN) was developed in [11]. This method improved the performance of classification with $93.65 \%$ specificity and $83.68 \%$ accuracy. Matthew et al. [12] developed a model for automatic exudate detection in retinal images from diabetic patients. They presented a series of experiments on feature selection and exudates classification using KNN and SVM classifiers. Authors [13] reviews algorithm for extracting features based on blood vessel area, exudes, hemorrhages, microaneurysms and texture from digital fundus images for the automated detection of DR. Varun et al. [14] relied on the development and validation of a deep learning algorithm for automated detection of diabetic retinopathy and diabetic macular edema in retinal fundus photographs. Paper [15] used ensemble of machine learning classifying algorithms on features extracted from output of different retinal image to give decision about the presence of diabetic retinopathy and achieved $75.32 \%$ accuracy by applying Neural Network (NNET) classifier. A fully automated DR screening system based on Morphological Component Analysis (MCA) to differentiate between normal and pathological retinal structures was considered in Imani et al. [16]. A SVM classifier was employed to differentiate between normal and abnormal retinal images and achieved $92.01 \%$ sensitivity and $95.45 \%$ specificity on the Messidor dataset. Goh et al. [17] filtered the retinal healthy images from DR based on the result of exudates detection using the features of local sub-images as the input to multiple classifiers. The experimental results demonstrated that by applying ensemble classifiers they obtained an accuracy of $92 \%$ in detecting normal images and $91 \%$ in detecting abnormal images over 1000 retina images. A review of recent development on detection methods for the diagnosis of DR was presented in [18] that was developed by various computational intelligence and image processing techniques. A combination of fuzzy image processing techniques, the circular hough transform and several feature extraction methods were proposed by Rahim et al. [19] for automatic screening and classification of diabetic retinopathy and maculopathy. They reached the value of sensitivity, specificity and accuracy of $92.45 \%, 93.62 \%$ and $93 \%$ respectively by using radial basis function SVM classifier. Manjaramkar et al. [20] presented a simple, efficient and real-time method for the segmentation and detection of microaneurysm (MA) which are the clinical signs of DR. For this, they used a novel set of features based on statistics of geometrical properties of connected regions and attained the best performance on perimage evaluation on DIARETDB1 dataset with sensitivity of $88.09 \%$ at $92.65 \%$ specificity.

In our work, we have developed a Computer Aided Decision Support System (CADSS) for automated DR detection and classification based on statistical texture features. Our method is promising and effective over previous state-of-the-art approaches and thus reduces the number of false positives. To identify DR in the retinal images, this study utilizes GLCM and GLRLM as a feature extraction technique. The GLCM and GLRLM capacities describe the composition of an image by computing how regularly combines of the pixel with particular qualities and in a predefined spatial relationship happen in an image. The features are learned in different scales to provide scale-invariant features through GLCM and GLRLM then each image in the frame is classified as abnormal (PDR and NPDR) or normal (normal retinal image). This research represents a possible improvement in the detection and classification of diabetic retinopathy.

The remainder of the paper is as follows: a review of the necessary background required to effectively implement our algorithm is presented in section II. After that, application of the proposed system as well as experimental results is described in section III and section IV. Finally we draw a conclusion in section V.

\section{Background}

\section{A. Acquisition of retinal image}

A large number of publicly available DR datasets is a crucial factor for this purpose. However, the performance of any Computer-Aided Design (CAD) system depends on the training dataset [21], this proposed system utilizes more than 600 standard color fundus images from different sources. Most fundus photographs used in this study were DR $(>78 \%$ ) images and only $21 \%$ were normal retinal images. The sample dataset of two categories of DR along with normal retinal image is shown in Fig. 1. 


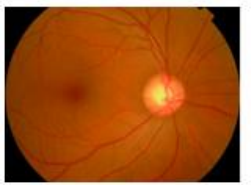

(a)

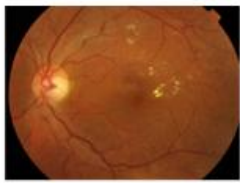

(b)

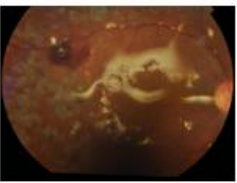

(c)

Fig. 1: Sample dataset: (a) normal retina, (b) non-proliferative DR and (c) proliferative DR

Important sources of the database include Indian Diabetic Retinopathy Image Dataset (https://idrid.grandchallenge.org/Data/) and Kaggle dataset (https://www.kaggle.com/c/diabetic retinopathy-detection). Table 1 shows the distribution of dataset used in the proposed method.

\section{B. Pre-processing}

An input image is loaded in the computer to find the possible categories of retinal images using the proposed intelligent system. For better and faster calculation, all the input image is resized into uniform size (565*375) image.

Table 1. Our dataset: the distribution of retinal images used in our proposed system

\begin{tabular}{lcc}
\hline Retinal image type & $\begin{array}{l}\text { Short } \\
\text { name }\end{array}$ & $\begin{array}{c}\text { Number of } \\
\text { images }\end{array}$ \\
\hline $\begin{array}{l}\text { Non-Proliferative Diabetic } \\
\text { Retinopathy }\end{array}$ & NPDR & 167 \\
\hline $\begin{array}{l}\text { Proliferative Diabetic } \\
\text { Retinopathy }\end{array}$ & PDR & 341 \\
\hline Normal Image & - & 136 \\
\hline
\end{tabular}

\section{Blood vessels extraction}

In our proposed method, we have used kirsch's template for the extraction of blood vessels from the pre-processed retinal image. Kirsch's template technique uses a single mask of size $3 \times 3$ and rotates at $45^{\circ}$ increments through each of the eight directions (south, east, north, west, northeast, southeast, southwest and northwest) to detect the edge as shown in Fig. 2. It can set and reset the thresholds values to find the most appropriate edges in the images [22]. The details procedure can be found in [23]. Fig. 3 demonstrates the vessel extraction procedure with Kirsch's template technique.

\begin{tabular}{|c|c|c|}
\hline 5 & -3 & -3 \\
\hline 5 & 0 & -3 \\
\hline 5 & -3 & -3 \\
\hline \multicolumn{3}{|c|}{$0^{\circ}$} \\
\hline-3 & -3 & -3 \\
\hline-3 & 0 & -3 \\
\hline 5 & 5 & 5 \\
\hline
\end{tabular}

\begin{tabular}{|c|c|c|}
\hline-3 & -3 & 5 \\
\hline-3 & 0 & 5 \\
\hline-3 & -3 & 5 \\
\hline \multicolumn{3}{|c|}{$45^{\circ}$} \\
\hline 5 & 5 & 5 \\
\hline-3 & 0 & -3 \\
\hline-3 & -3 & -3 \\
\hline
\end{tabular}

\begin{tabular}{|c|c|c|}
\hline-3 & -3 & -3 \\
\hline 5 & 0 & -3 \\
\hline 5 & 5 & -3 \\
\hline \multicolumn{3}{|c|}{$90^{\circ}$} \\
\hline-3 & -3 & -3 \\
\hline-3 & 0 & 5 \\
\hline-3 & 5 & 5 \\
\hline
\end{tabular}

\begin{tabular}{|c|c|c|}
\hline-3 & 5 & 5 \\
\hline-3 & 0 & 5 \\
\hline-3 & -3 & -3 \\
\hline \multicolumn{3}{|c|}{$135^{\circ}$} \\
\hline 5 & 5 & -3 \\
\hline 5 & 0 & -3 \\
\hline-3 & -3 & -3 \\
\hline
\end{tabular}

Fig. 2. Kirsch's convolution kernels
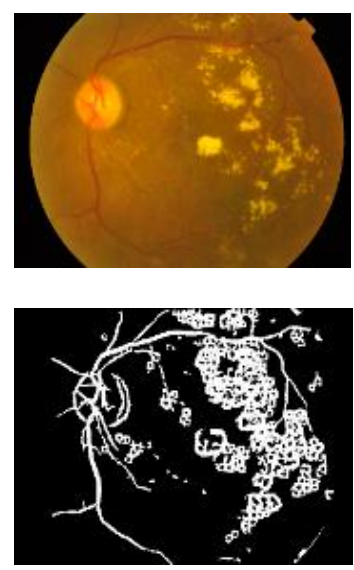

Fig.3. Resultant image after segmentation: (a) the first row shows the image of PDR, NPDR and normal retinal image respectively and (b) the last row indicates the blood vessel extraction results 


\section{Feature extraction}

In feature extraction module, statistical texture features are applied on the segmented image. A feature vector is created by using a large number of training images.

The Gray Level Co-occurrence Matrix (GLCM) also known as Spatial Gray Level Dependence Matrix (SGLDM) is a way to gain second-order statistical texture feature. The GLCM method analyses the distribution of gray level pixel pairs. It provides better results where the textures are visually easily separable and are sensitive to the size of the texture samples being processed. Nine important GLCM features are selected as a second-order statistical texture feature.

$$
\begin{gathered}
\text { Contrast }=\sum_{n=0}^{\mathrm{G}-1} \mathrm{n}^{2}\left\{\sum_{\mathrm{i}=1}^{\mathrm{G}} \sum_{\mathrm{j}=1}^{\mathrm{G}} \mathrm{P}(\mathrm{i}, \mathrm{j})\right\},|\mathrm{i}-\mathrm{j}|=\mathrm{n} \\
\text { Correlation }=\sum_{\mathrm{i}=0}^{\mathrm{G}-1} \sum_{\mathrm{j}=0}^{\mathrm{G}-1} \frac{\{\mathrm{i} * j\} * \mathrm{p}(\mathrm{i}, \mathrm{j})-\left\{\mu_{\mathrm{x}^{*}} \mu_{\mathrm{y}}\right\}}{\sigma_{\mathrm{x}} \sigma_{\mathrm{y}}} \\
\text { Energy }=\sum_{\mathrm{i}=0}^{\mathrm{G}-1} \sum_{\mathrm{j}=0}^{\mathrm{G}-1}\{\mathrm{P}(\mathrm{i}, \mathrm{j})\}^{2} \\
\text { Entropy=- } \sum_{\mathrm{i}=0}^{\mathrm{G}-1} \sum_{\mathrm{j}=0}^{\mathrm{G}-1} \mathrm{P}(\mathrm{i}, \mathrm{j}) * \log (\mathrm{p}(\mathrm{i}, \mathrm{j})) \\
\text { Inverse Different Moment= } \sum_{\mathrm{i}=0}^{\mathrm{G}-1} \sum_{\mathrm{j}=0}^{\mathrm{G}-1} \frac{1}{1+(\mathrm{i}-\mathrm{j})^{2}} \mathrm{P}(\mathrm{i}, \mathrm{j}) \\
\text { Variance }=\sum_{\mathrm{i}=0}^{\mathrm{G}-1} \sum_{\mathrm{j}=0}^{\mathrm{G}-1}(\mathrm{i}-\mu)^{2} \mathrm{P}(\mathrm{i}, \mathrm{j}) \\
\text { Sum Average= } \sum_{\mathrm{i}=0}^{2 \mathrm{G}-2} \mathrm{i} \mathrm{P}_{\mathrm{x}+\mathrm{y}}(\mathrm{i}) \\
\text { Sum Entropy }=-\sum_{\mathrm{i}=0}^{2 \mathrm{G}-2} \mathrm{P}_{\mathrm{x}+\mathrm{y}}(\mathrm{i}) \log \left(\mathrm{P}_{\mathrm{x}+\mathrm{y}}(\mathrm{i})\right) \\
\text { Difference Entropy }=-\sum_{\mathrm{i}=0}^{\mathrm{G}-1} \mathrm{P}_{\mathrm{x}+\mathrm{y}}(\mathrm{i}) \log \left(\mathrm{P}_{\mathrm{x}+\mathrm{y}}(\mathrm{i})\right)
\end{gathered}
$$

Where, $\mathrm{G}$ is the number of gray levels used; $\mu$ is the mean value of $\mathrm{P} ; \mu_{\mathrm{x}}, \mu_{\mathrm{y}}, \partial_{\mathrm{x}}$ and $\partial_{\mathrm{y}}$ are the means and standard deviations of $\mathrm{P}_{\mathrm{x}}$ and $\mathrm{P}_{\mathrm{y}} ; \mathrm{P}_{\mathrm{x}}(\mathrm{i})$ is the ith entry in the marginal matrix obtained by summing rows of co-occurrence matrix, $\mathrm{P}(\mathrm{i}, \mathrm{j})$.

The Gary Level Run Length Matrix (GLRLM) has been considered for the description of higher-order statistical texture feature implemented within the segmented image. The GLRLM method quantifies gray level runs of consecutive pixels with the same gray level value, which are defined as the length in number of pixels. The run length features are the least efficient texture features. We consider seven run length matrix features.

$$
\begin{gathered}
\text { Short Run Emphasis }=\frac{1}{\mathrm{n}_{\mathrm{r}}} \sum_{\mathrm{i}=1}^{\mathrm{M}} \sum_{\mathrm{j}=1}^{\mathrm{N}} \frac{\mathrm{Q}(\mathrm{i}, \mathrm{j})}{\mathrm{j}^{2}} \\
\text { Long Run Emphasis }=\frac{1}{\mathrm{n}_{\mathrm{r}}} \sum_{\mathrm{i}=1}^{\mathrm{M}} \sum_{\mathrm{j}=1}^{\mathrm{N}} \mathrm{Q}(\mathrm{i}, \mathrm{j}) * \mathrm{j}^{2} \\
\text { Long Gray Level Emphasis }=\frac{1}{\mathrm{n}_{\mathrm{r}}} \sum_{\mathrm{i}=1}^{\mathrm{M}} \sum_{\mathrm{j}=1}^{\mathrm{N}} \frac{\mathrm{Q}(\mathrm{i}, \mathrm{j})}{\mathrm{i}^{2}} \\
\text { High Gray Level Emphasis }=\frac{1}{\mathrm{n}_{\mathrm{r}}} \sum_{\mathrm{i}=1}^{\mathrm{M}} \sum_{\mathrm{j}=1}^{\mathrm{N}} \mathrm{Q}(\mathrm{i}, \mathrm{j}) * \mathrm{i}^{2} \\
\text { Gray Level Non-uniformity }=\frac{1}{\mathrm{n}_{\mathrm{r}}} \sum_{\mathrm{i}=1}^{\mathrm{M}}\left(\sum_{\mathrm{j}=1}^{\mathrm{N}} \mathrm{Q}(\mathrm{i}, \mathrm{j})\right)^{2} \\
\text { Run Length Non-uniformity }=\frac{1}{\mathrm{n}_{\mathrm{r}}} \sum_{\mathrm{j}=1}^{\mathrm{N}}\left(\sum_{\mathrm{i}=1}^{\mathrm{M}} \mathrm{Q}(\mathrm{i}, \mathrm{j})\right)^{2} \\
\text { Run Percentage }=-\frac{\mathrm{n}_{\mathrm{p}}}{\mathrm{n}_{\mathrm{r}}}
\end{gathered}
$$

Where $P(i, j)$ is the run-length matrix, $n_{r}$ is the total number of runs and $n_{p}$ is the number of pixels. 


\section{E. Classification}

This module handles the classification of feature vector into three classes (Normal, NPDR and PDR). The whole feature vector is divided into two modes: training and testing modes by using K-fold cross-validation [24]. In training mode, GLCM and GLRLM features are extracted from segmented images. The input feature vector consists of 16 different features which are combined together for training with three different classifiers namely KNN [25], SVM [26] and RF [27]. Our proposed system first detects whether the unknown sample contains DR or not. If it contains DR then the unknown sample is classified as NPDR or PDR and it goes to the post-processing module. If it does not contain DR then the unknown sample is classified as normal.

\section{F. Post-processing}

The outcome of the classification module is used to a produce new image on which possible types of diabetic retinopathy are appropriately labeled. An illustration of this technique shown in Fig. 4.

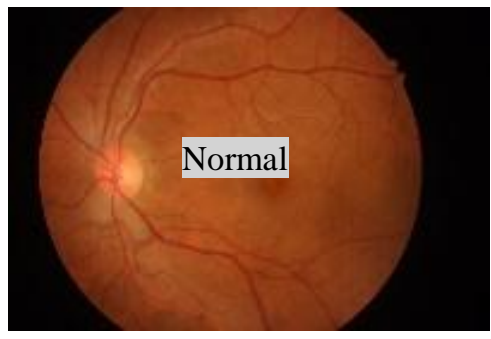

(a)

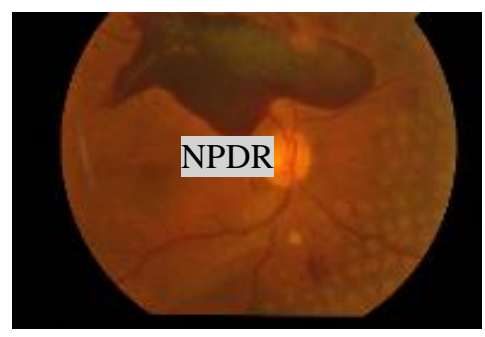

(b)

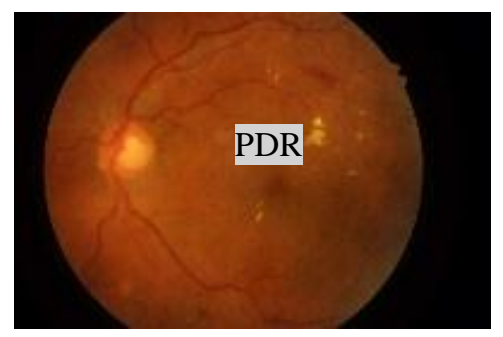

(c)

Fig. 4. System's output after post-processing: (a) normal retinal image, (b) NPDR image, and (c) PDR image

\section{Methodology}

Fig. 5 shows the methodology of our proposed system. The proposed methodology is summarized as follows.

\section{A. Proposed Algorithm}

1. Capture the digital fundus image as input image.

2. Pre-process all input image into a uniform size.

3. Apply Kirsch's template technique to extract blood vessels from the preprocessed image.

4. Execute second-order and higher-order statistical texture feature algorithm on the segmented image found from step 3.

5. Generate a Feature Vector (FV) for each training image.

6. Implement three classifiers (SVM, KNN, and RF) to test the train image for classification and evaluate the performance of the results.

\section{Experiment simulation and Result Analysis}

To assess efficiency of the proposed system, we evaluated four performance metrics for each class: sensitivity, precision, F1-score and accuracy. Sensitivity also known as recall is the ratio of correctly predicted positives cases to the all observations in actual class. The precision metric indicates the correct positive outcomes out of all the positive outcomes. The accuracy of a classifier is simply the ratio of correctly predicted class to total class. F1-score is estimated by applying the weighted average over precision and recall. In case we have an uneven class proportion, F1-score is generally more valuable than precision because it takes both false positives and false negatives into account. Our main purpose is to construct a model that classifies diabetic retinopathy as accurately as possible. We used DR database with 644 images for the evaluation, where the entire dataset is distributed within the training and test dataset. The performance of detecting and classifying DR using three different classifiers are shown in Table 2, Table 3, Table 4, and Table 5 respectively. Finally, the sensitivity, precision, F1-score, and accuracy scores across the three different classes of each classifier is shown in Table 6. From Table 6 to Table 7, "Sn" is for sensitivity, "Pre" for precision, and "Acc" for accuracy. 


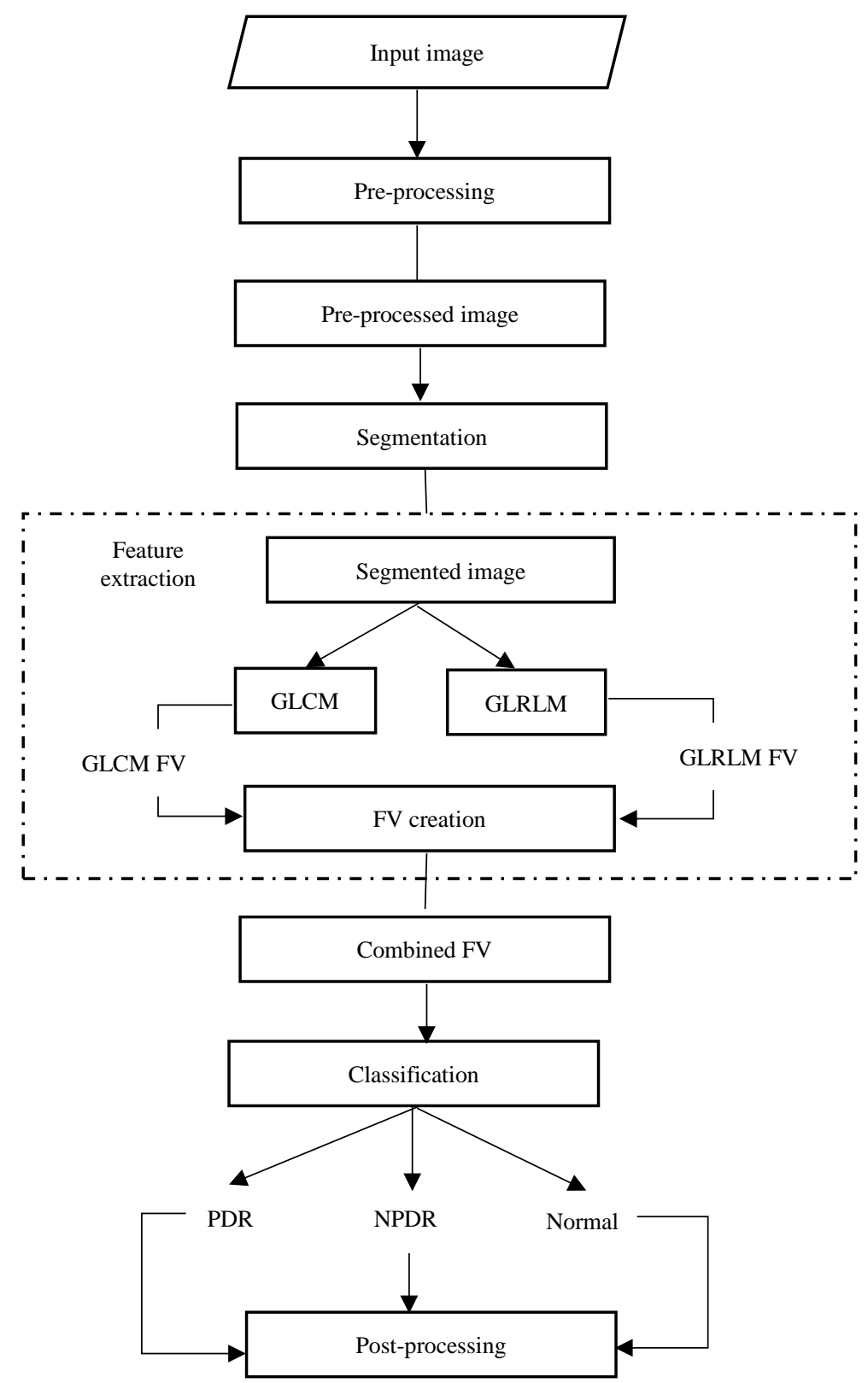

Fig. 5. Detailed diagram of the proposed methodology

Table 2. Confusion matrix of our proposed system using SVM classifier

\begin{tabular}{|c|c|c|c|}
\hline & PDR & NPDR & Normal \\
\hline PDR & 326 & 11 & 4 \\
\hline NPDR & 19 & 136 & 12 \\
\hline Normal & 4 & 3 & 129 \\
\hline
\end{tabular}

Table 3. Confusion matrix of our proposed system using $\mathrm{KNN}(\mathrm{K}=3)$ classifier

\begin{tabular}{|c|c|c|c|}
\hline & PDR & NPDR & Normal \\
\hline PDR & 302 & 23 & 16 \\
\hline NPDR & 21 & 127 & 19 \\
\hline Normal & 18 & 14 & 104 \\
\hline
\end{tabular}

Table 4. Confusion matrix of our proposed system using $\mathrm{KNN}(\mathrm{K}=5)$ classifier

\begin{tabular}{|c|c|c|c|}
\hline & PDR & NPDR & Normal \\
\hline PDR & 308 & 17 & 16 \\
\hline NPDR & 21 & 136 & 10 \\
\hline Normal & 18 & 19 & 99 \\
\hline
\end{tabular}


Table 5. Confusion matrix of our proposed system using RF classifier

\begin{tabular}{|c|c|c|c|}
\hline & PDR & NPDR & Normal \\
\hline PDR & 333 & 5 & 3 \\
\hline NPDR & 6 & 149 & 12 \\
\hline Normal & 2 & 3 & 131 \\
\hline
\end{tabular}

From the confusion matrix of Table 2, Table 3, Table 4 and Table 5 we see that the random forest classifier made a correct prediction of 333 PDR, 149 NPDR and 131 Normal images out of 341 PDR, 167 PDR and 136 Normal images which is higher than the other two classifiers. For performance evaluation parameters of each transform individually, where we can see that using random forest classifier, the highest sensitivity is in PDR images which comes out to be $98 \%$ and the worst sensitivity is $90 \%$ which is in normal images. Similarly both the precision and F1-score we get the highest rate of $98 \%$ in PDR images. Our method attains the highest accuracy of $97.52 \%$ for the detection of PDR images. Table 7 contains the result of weighted measurement of each classifier.

Table 6. Sensitivity, precision, F1-score and accuracy of each classifier

\begin{tabular}{|c|c|c|c|c|c|c|c|c|c|c|c|c|c|c|c|c|}
\hline \multirow{2}{*}{ Image Type } & \multicolumn{4}{|c|}{ SVM } & \multicolumn{4}{|c|}{$\mathrm{KNN}(\mathrm{K}=3)$} & \multicolumn{4}{|c|}{$\mathrm{KNN}(\mathrm{K}=5)$} & \multicolumn{4}{|c|}{$\mathrm{RF}$} \\
\hline & $\mathrm{Sn}$ & Pre & F1 & Acc & Sn & Pre & $\mathrm{F} 1$ & Acc & $\mathrm{Sn}$ & Pre & F1 & Acc & Sn & Pre & F1 & Acc \\
\hline PDR & 93 & 96 & 94 & 94.1 & 89 & 89 & 89 & 87.89 & 89 & 90 & 90 & 88.82 & 98 & 98 & 98 & 97.52 \\
\hline NPDR & 91 & 81 & 86 & 93.01 & 77 & 76 & 77 & 88.04 & 79 & 81 & 80 & 89.6 & 95 & 89 & 92 & 95.92 \\
\hline Normal & 89 & 95 & 92 & 96.43 & 75 & 76 & 76 & 89.6 & 79 & 73 & 76 & 90.22 & 90 & 96 & 93 & 96.89 \\
\hline
\end{tabular}

Table 7. Weighted measure of each classifier

\begin{tabular}{ccccc}
\hline \multirow{2}{*}{ Name of the classifier } & \multicolumn{4}{c}{ Weighted Measure } \\
\cline { 2 - 5 } & Sn & Pre & F1-score & Acc \\
\hline SVM & 91.63 & 91.89 & 91.35 & 94.30 \\
\hline KNN $(\mathrm{K}=3)$ & 82.93 & 82.88 & 83.14 & 88.29 \\
\hline KNN $(\mathrm{K}=5)$ & 83.66 & 84.07 & 84.45 & 89.31 \\
\hline RF & 95.53 & 96.45 & 95.38 & 95.19 \\
\hline
\end{tabular}

Based on Table 7, the result obtained that the weighted accuracy value of the proposed method reached $95.19 \%$ using RF classifier. Sensitivity, precision and F1-score of the proposed method respectively $95.53 \%, 96.45 \%$ and 95.38\%. A graphical representation of the performance metrics of each classifier is shown in Fig. 6.

Table 8. Performance comparison among different methods

\begin{tabular}{|c|c|c|c|}
\hline Authors & Methodology & Dataset & Accuracy \\
\hline Khademi et al. [8] & $\begin{array}{c}\text { Shift-invariant discrete } \\
\text { wavelet transform }\end{array}$ & 86 & $82.2 \%$ \\
\hline Neto et al. [9] & $\begin{array}{c}\text { Unsupervised coarse-to-fine } \\
\text { algorithm }\end{array}$ & 60 & $87 \%$ \\
\hline Sarathi et al. [10] & Ellipse fitting & 63 & $92 \%$ \\
\hline Garcia et al. [11] & CNN & 35,126 & $83.68 \%$ \\
\hline Rahim et al. [19] & $\begin{array}{c}\text { Fuzzy image processing } \\
\text { techniques }\end{array}$ & 990 & $93 \%$ \\
\hline Manjaramkar et al. [20] & Statistical geometric features & 89 & $88.09 \%$ \\
\hline Proposed Method & Statistical texture features & 644 & $95.19 \%$ \\
\hline
\end{tabular}

Table 8 shows the comparison of our proposed method with existing approaches. We compare the methods focused on the detection and classification of two main types of DR.

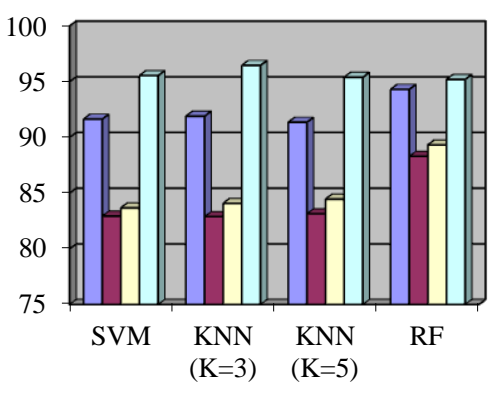

$$
\text { 口Accuracy 口F1-score 口Precision aSensitivity }
$$

Fig. 6.Graphical performance of the model for different evaluation criteria 


\section{Discussion}

Retinal color photography is an efficient screening method for diagnosing diabetic retinopathy because of it's faster with easier acquisition, storage and transmission of retinal images. Most of the middle and lower-income countries require a cost-effective retinal imaging framework for regular diabetic retinopathy screening [28]. Therefore, an automatic system for the detection and classification of diabetic retinopathy using statistical texture features has been created. The system proposes a combination of retinal blood vessels segmentation, followed by the feature extraction and, finally, the classification with some machine learning algorithms. We incorporate both second-order and higherorder statistical texture feature as a feature extraction tool. As texture features can be extracted using several methods such as structural, statistical, model-based and transform information, we consider statistical methods. According to non-deterministic properties, statistical methods describe the texture indirectly. We have used 10-fold cross-validation for splitting the whole dataset to protect against overfitting. To extract statistical texture descriptors from vessel images, the GLCM and GLRLM features have been used that can demonstrate sound performance in image classification of different categories. An ideal screening test can be measured by its sensitivity i.e., high probability of detecting disease. The experimental results demonstrate that the three classifiers are able to identify well both categories and particularly random forest classifier performed better in most of the cases than the other two classifiers. The sensitivity of retinal images using RF classifier was high (98\%) for PDR and for NPDR was also high (95\%). Based on the offered results, it can be seen that the sensitivity for normal cases is slightly low $(90 \%)$. The explanation may be the functionality of kirsch's template technique which was used for the detection of blood vessels. Some of the blood vessels were not detected by this technique.

\section{Conclusion and Future works}

A large number of diabetic patients and the incidence of DR among them have promoted a great development in automatic DR diagnosing systems. In this study, we developed a computer-aided DR detection and classification system form color fundus images in order to reduce the workload of ophthalmologists to detect DR at the early stages which may go undetected and evolve into blindness. Weighted value of sensitivity, precision, F1-score and accuracy of the suggested method respectively $95.53 \% 96.45 \%, 95.38 \%$, and $95.19 \%$ which shows that the proposed method can detect and classify DR well. In the future, these methods should be tested on integrating a larger dataset with high-resolution images. Finally, we will add more classes of non-proliferative diabetic retinopathy viz. low, medium, severe condition. It will enable the patients to know their condition with better accuracy.

\section{Acknowledgment}

We are very grateful to Khwaja Yunus Ali Medical College and Hospital, Bangladesh for valuable supports and suggestions.

\section{References}

[1] D. Fong, L. Aiello, T. Gardner, G. King, G. Blankenship, et al. "Retinopathy in Diabetes". Diabetes Care. Vol. 27. 2004. pp. 584-587.

[2] D. Browning, "Diabetic Retinopathy: Evidence Based Management”, 1st ed. Ed. Springer, New York, USA, 2010 , pp. 31 -61.

[3] BoserB ,Guyon I.G,Vapnik V., "A Training Algorithm for Optimal Margin Classifiers", Proc. Fifth Ann. Workshop Computational Learning Theory, pp. 144-152, 1992.

[4] Nathan Silberman, Kristy Ahlrich, Rob Fergus \& Lakshminarayanan Subramanian (2010), "Association for the Advancement of Artificial Intelligence", October, 2013.

[5] Fong. D. S. , L. Aiello, T. W. Gardner, G. L. King, G. Blankenship, J. D. Cavallerano, F. L. Ferris \& R. Klein, (2003),"Diabetic Retinopathy", Diabetes Care, Vol. 26, pp. 226-229.

[6] Velázquez-González, Jesús Salvador, Rosales-Silva, Alberto Jorge, Gallegos-Funes, Francisco Javier, \& Guzmán-Bárcenas, Guadalupe de Jesús. (2015), "Detection and Classification of Non-Proliferative Diabetic Retinopathy using a Back-Propagation Neural Network", Revista Facultad de Ingeniería Universidad de Antioquia, (74), 70-85.

[7] L. Daniel Maxim, Ron Niebo, "Screening tests: a review with examples", Inhal Toxicol. 2014 Nov; 26(13): 811-828.

[8] Khademi and S. Krishnan, "Shift-invariant discrete wavelet transform analysis for retinal image classification," Medical and Biological Engineering and Computing, vol. 45, issue 12, pp. 1211-1222, 2007.

[9] Neto, L.C.; Ramalho, G.L.; Neto, J.F.R.; Veras, R.M.; Medeiros, F.N., "An unsupervised coarse-to-fine algorithm for blood vessel segmentation in fundus images”, Expert System with Application, Vol. 78, pp. 182-192, 2017.

[10] Sarathi, M.P. Dutta, M.K. Singh, A. Travieso, C.M., "Blood vessel in painting based technique for efficient localization and segmentation of optic disc in digital fundus images", Biomedical Signal Processing, Vol. 25, pp. 108-117, 2016.

[11] García G., Gallardo J., Mauricio A., López J., Del Carpio C., "Detection of diabetic retinopathy based on a convolutional neural network using retinal fundus images", In Lecture Notes in Computer Science, Proceedings of the Artificial Neural Networks and Machine Learning-ICANN, vol. 10614, pp. 635-642, 2017. 
[12] Matthew N. Dailey, Bunyarit Uyyanonvara, Sarah Barman, Tom Williamson, Khine Thet Nwe, "Machine learning approach to automatic exudate detection in retinal images from diabetic patients", Tailor and Francis online Volume 57, 2010.

[13] Rajendra Acharya U., E. Y. Ng, Kwan-Hoong Ng, "Algorithms for the Automated Detection of Diabetic Retinopathy Using Digital Fundus Images", Journal of Medical Systems, Volume 36, No. 1, February 2012, doi.org/10.1007/s10916-010-9454-7.

[14] Varun G., Lily P., Mark C., "Development and validation of a deep learning Algorithm for Detection of Diabetic Retinopathy", November 2016, JAMA The Journal of the American Medical Association 316(22), doi: 10.1001/jama.2016.17216

[15] Maliha M., Tareque A. and Roy S. S. 2018 Diabetic Retinopathy Detection Using Machine Learning

[16] Imani, E., Pourreza, H.R., Banaee, T, "Fully automated diabetic retinopathy screening using morphological component analysis", Comput. Med. Imaging Graph. March 2015, 43, 78-88, doi: 10.1016/j.compmedimag.2015.03.004

[17] Goh, J., Tang, L., Saleh, G., Al Turk, L.; Fu, Y., Browne, A, "Filtering normal retinal images for diabetic retinopathy screening using multiple classifiers", In Proceedings of the 9th International Conference on Information Technology and Applications in Biomedicine, December 2009, doi: 10.1109/ITAB.2009.5394392

[18] Qureshi, I., Ma, J., \& Abbas, Q. (2019), "Recent Development on Detection Methods for the Diagnosis of Diabetic Retinopathy", Symmetry, 11(6), 749. doi:10.3390/sym11060749

[19] Rahim, S. S., Palade, V., Shuttleworth, J., \& Jayne, C. (2016), "Automatic screening and classification of diabetic retinopathy and maculopathy using fuzzy image processing”, Brain Informatics, 3(4), 249-267, doi: 10.1007/s40708-016-0045-3.

[20] Manjaramkar, A., \& Kokare, M. (2017), "Statistical Geometrical Features for Microaneurysm Detection”, Journal of Digital Imaging, 31(2), 224-234, doi: 10.1007/s10278-017-0008-0.

[21] Bin Zheng, PhD, Xingwei Wang, PhD, Dror Lederman, PhD, Jun Tan, "Computer-Aided Detection - The Effect of Training Databases on Detection of Subtle Breast Masses", 2010 Nov, 17(11): 1401-1408.

[22] H.S. Bhadauria (2013), "Vessels Extraction from Retinal Images", IOSR J. Electron. Commun. Eng. $679-82$

[23] T. Jemima Jebaseeli, C. Anand Deva Durai,1 and J. Dinesh Peter, "Extraction of Retinal Blood Vessels on Fundus Images by Kirsch's Template and Fuzzy C-Means”, J Med Phys, 2019 Jan-Mar; 44(1): 21-26, doi: 10.4103/jmp.JMP_51_18

[24] Good, P.I. (2006), "Resampling methods: A practical guide to data analysis", 3rd Edition, Birkhauser

[25] Sapiah Binti Sakri, Nuraini Binti Abdul Rashid, and Zuhaira Muhammad Zain, "Particle Swarm Optimization Feature Selection for Breast Cancer Recurrence Prediction", Special Section on Big Data Learning and Discovery, IEEE Access, DOI 10.1109/ACCESS.2018.2843443.

[26] Gandhi M, Dhanasekaran R, "Diagnosis of Diabetic Retinopathy Using Morphological Process and SVM Classifier", IEEE International conference on Communication and Signal Processing, 2013, India pp: 873-877.

[27] Cuong Nguyen, Yong Wang, Ha Nam Nguyen, "Random forest classifier combined with feature selection for breast cancer diagnosis and prognostic", J. Biomedical Science and Engineering, 2013, 6, 551-560.

[28] Javit JC. Cost-savings associated with detection and treatment of diabetic eye disease. PharmacoEconomics 1995; 8:33-39.

\section{Authors' Profiles}

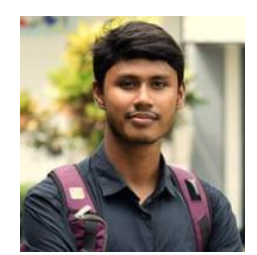

Md. Rahat Khan is currently pursuing his B.Sc (Engg.) in Computer Science and Engineering from Khwaja Yunus Ali university, Bangladesh. His research interest includes image and signal processing, machine learning, neural networks, and bioinformatics.

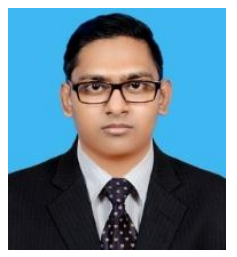

A. S. M. Shafi received the M.Sc. (Engg.) degree in Computer Science and Engineering (CSE) in 2016 from the University of Mawlana Bhashani Science and Technology (MBSTU), Bangladesh. He is currently working as a lecturer of Computer Science and Engineering department with the University of Khwaja Yunus Ali, Bangladesh. His area of research interests are medical image processing, digital image processing, deep learning, neural networks, computer vision and data mining

How to cite this paper: Md. Rahat Khan, A. S. M. Shafi, " Statistical Texture Features Based Automatic Detection and Classification of Diabetic Retinopathy", International Journal of Image, Graphics and Signal Processing(IJIGSP), Vol.13, No.2, pp. 53-61, 2021.DOI: $10.5815 /$ ijigsp.2021.02.05 